OPEN ACCESS

Edited by:

Pingyu Zhou,

Tongji University, China

Reviewed by:

Meiping Ye,

Shanghai Skin Disease Hospital, China

Lorenzo Giacani,

University of Washington,

United States

*Correspondence:

Tian-Ci Yang

yangtianci@xmu.edu.cn

Specialty section:

This article was submitted to

Microbial Immunology,

a section of the journal

Frontiers in Immunology

Received: 19 November 2021 Accepted: 08 February 2022

Published: 28 February 2022

Citation:

$X u Q-Y$, Wang $Y-J$, Lin L-R, Liu L-L and Yang T-C (2022) The Outer Membrane Lipoprotein Tp0136 Stimulates Human Platelet Activation and Aggregation Through PAR1 to Enhance $G_{d} / G_{i}$ Signaling.

Front. Immunol. 13:818151. doi: 10.3389/fimmu.2022.818151

\section{The Outer Membrane Lipoprotein Tp0136 Stimulates Human Platelet Activation and Aggregation Through PAR1 to Enhance $G_{\mathbf{q}} / G_{\mathbf{i}}$ Signaling}

\author{
Qiu-Yan Xu ${ }^{1,2}$, Yong-Jing Wang ${ }^{1,2}$, Li-Rong Lin ${ }^{1,2}$, Li-Li Liu ${ }^{1,2}$ and Tian-Ci Yang ${ }^{1,2 *}$ \\ ${ }^{1}$ Center of Clinical Laboratory, Zhongshan Hospital of Xiamen University, School of Medicine, Xiamen University, \\ Xiamen, China, 2 Institute of Infectious Disease, School of Medicine, Xiamen University, Xiamen, China
}

Background: Chancre self-healing, a typical clinical phenomenon of primary syphilis, is essentially wound healing. The first response to a wound is constriction of the injured blood vessels and activation of platelets to form a fibrin clot. However, the role of Treponema pallidum in platelet activation and clot formation remains unclear.

Objectives: We aimed to elucidate the role of the outer membrane Treponema pallidum lipoprotein Tp0136 in human platelet activation and aggregation and explore the related mechanism.

Methods: A series of experiments were performed to assess the effects of Tp0136 on human platelet activation and aggregation in vitro. The effect of Tp0136 on platelet receptors was studied by detecting PAR1 protein levels and studying related receptor sites. The involvement of the $G_{q} / G_{i}$ signaling pathway downstream of PAR1 was explored.

Results: Tp0136 significantly accelerated the formation of human platelet clots as well as platelet adhesion to and diffusion on fibrinogen to promote platelet aggregation. Tp0136 also potentiated P-selectin expression and PF4 release to promote platelet activation and downregulated PAR1 expression. The activation and aggregation induced by Tp0136 were reverted by the specific PAR1 antagonist RWJ56110 and the human PAR1 antibody. In addition, Tp0136 significantly enhanced $G_{q}$ and $G_{i}$ signaling activation, thereby triggering p38 phosphorylation and Akt-PI3K activation, increasing the release of intraplatelet $\mathrm{Ca}^{2+}$ and attenuating the release of cytosolic cAMP. Furthermore, the specific PAR1 antagonist RWJ56110 significantly suppressed $G_{a}$ and $G_{i}$ signaling activation.

Conclusions: Our results showed that the Treponema pallidum Tp0136 protein stimulated human platelet activation and aggregation by downregulating PAR1 and triggered PAR1-dependent $G_{q}$ and $G_{i}$ pathway activation. These findings may contribute to our understanding of the self-healing of chancroid in early syphilis.

Keywords: Tp0136, platelet activation, platelet aggregation, protease-activated receptor $1, G_{q}, G_{1}$ 


\section{HIGHLIGHTS}

1. Tp0136 enhances platelet activation and aggregation by downregulating PAR1.

2. Tp0136-downregulated PAR1 selectively stimulates PAR1dependent $G_{q}$ and $G_{i}$ pathway activation.

\section{INTRODUCTION}

Syphilis, a chronic multistage disease punctuated by asymptomatic periods of latency, is caused by the spirochete Treponema pallidum subsp. pallidum (hereafter T. pallidum) and is primarily transmitted sexually or vertically during pregnancy (1). Syphilis is clinically manifested when spirochetes replicating at the site of inoculation induce a local inflammatory response sufficient to generate a papule that subsequently ulcerates, forming a chancre; chancres are the defining lesions of primary syphilis and are typically painless and resolve spontaneously (2). Thus far, our understanding of the mechanism of chancre self-healing in syphilis is limited.

Wound healing is one of the most complex processes in the human body, involving the spatiotemporal synchronization of multiple cell types with different roles in the phases of hemostasis, inflammation, growth, reepithelialization, and remodeling (3). The first reaction to a wound is constriction of the injured blood vessels and activation of platelets that adhere to the damaged site and aggregate to form a fibrin clot, resulting in the early stabilization of platelet thrombi that thereby initiate hemostasis $(4,5)$. Human platelets express protease-activated receptor 1 (PAR1), the prototypical member of the G-proteincoupled receptor family, which is activated by a variety of proteases (6). The activation of PAR1 is sufficient to trigger platelet secretion and aggregation (7), and PAR1 can couple with members of the $G_{q}, G_{12 / 13}$, and $G_{i}$ families to impact a substantial of signaling pathway networks (8).

Tp0136, an outer membrane lipoprotein of T. pallidum, is also an adhesin that is predicted to bind to different host cells and thereby mediate the colonization of $T$. pallidum in different tissues during infection $(9,10)$. Our previous study found that Tp0136 promoted the migration and proliferation of fibroblasts (11) and microvascular endothelial cells (12), which could contribute to the mechanism of chancre self-healing in syphilis. In addition, high titers of anti-Tp0136 antibodies promoted the infiltration of inflammatory cells into local lesions and intensified tissue damage, thus delaying wound healing (13). While platelets have been shown to be a $T$. pallidum target (14), whether $T$. pallidum activates platelets through Tp0136 and promotes platelet aggregation to mediate the self-healing of chancre remains unclear. In the current study, we performed a series of in vitro experiments to elucidate the effect of Tp0136 on platelet activation and aggregation and analyzed PAR1 receptors and subsequent signaling pathways that involved in this process.

\section{MATERIALS AND METHODS}

\section{Preparation of the Tp0136 Protein and Removed Endotoxin}

Full-length Tp0136 was directly cloned into the pEXP-5-CT vector, and the Tp0136-His-Tag protein was purified by affinity chromatography using Ni-NTA as described previously (12). Endotoxin was removed from the recombinant Tp0136 protein with an EtEraser ${ }^{\text {TM }}$ Endotoxin Removal Kit (Chinese Horseshoe Crab Reagent Manufactory, Ltd., Xiamen, China). Tachypleus amebocyte lysate (Chinese Horseshoe Crab Reagent Manufactory, Ltd., Xiamen, China) was used to detect endotoxin in the Tp0136 preparation, which was found to have less than 0.05 endotoxin units (EUs)/mL. A cytotoxicity assay was performed to evaluate the effect of Tp0136 on endothelial cells viability using a lactate dehydrogenase kit (NEOBIOSCIENCE Biotechnology Co., Ltd. Beijing, China). The results showed no significant cytotoxicity in Tp0136-treated cells.

\section{Preparation of Human Platelets}

Platelets from healthy volunteers were separated by the differential centrifugation of whole blood in anticoagulation tubes containing $3.8 \%$ sodium citrate as previously described $(15,16)$. Platelet-rich plasma (PRP) was separated from the plasma samples and centrifuged at $180 \mathrm{~g}$ for 15 minutes at room temperature. The samples were then centrifuged at $800 \mathrm{~g}$ for 20 minutes to obtain the platelet precipitates, which were then resuspended in modified Tyrode's solution (Solarbio, Beijing, China) and diluted to $2.0-3.0 \times 10^{8} / \mathrm{mL}$ for the following experiments. The studies involving human participants were reviewed and approved by the Ethics Committee of Zhongshan Hospital, Xiamen University. All volunteers provided written informed consent in accordance with the Declaration of Helsinki.

\section{Assessment of Platelet Adhesion and Spreading}

Platelet adhesion assays were performed according to Boncler et al. (15). Samples in 96-well microplates coated with $2 \mathrm{mg} / \mathrm{mL}$ fibrinogen $\left(4^{\circ} \mathrm{C}\right.$, overnight) were blocked with $0.2 \%$ bovine serum albumin $\left(1\right.$ hour, $\left.37^{\circ} \mathrm{C}\right)$. The $\mathrm{PRP}$ samples were incubated for 15 minutes with thrombin $(0.5 \mathrm{U} / \mathrm{mL})$, Tp0136 (10 $\mu \mathrm{g} / \mathrm{mL}), \operatorname{Tp} 17(10 \mu \mathrm{g} / \mathrm{mL})$ or phosphate-buffered saline (PBS) in the absence or presence of different antagonists or agonists. Then, $50 \mu \mathrm{L}$ aliquots of PRP were added to the wells for 1 hour at $37^{\circ} \mathrm{C}$. After washing, the wells were filled with a substrate solution and incubated for 1 hour. To estimate "total platelet adhesion", the PRP samples were mixed with the substrate solution and added to the uncoated wells. Plateletdeficient plasma was used as a blank control. Sodium hydroxide (2 M) was added to stop the enzymatic reaction, and the absorbance at $405 \mathrm{~nm}$ was read using a microplate analyzer (Thermo Scientific Multiskan FC, USA). The percentage of adherent platelets was calculated using the following formula: (sample-blank)/(total-blank) $\times 100$. 
Platelet cytoskeleton staining assays were performed as described in a previous study (17). PRP samples were treated with thrombin $(0.5 \mathrm{U} / \mathrm{mL}), \mathrm{Tp} 0136(10 \mu \mathrm{g} / \mathrm{mL}), \mathrm{Tp} 17(10 \mu \mathrm{g} /$ $\mathrm{mL}$ ) or PBS with or without RWJ56110 (a PAR1 antagonist) (1 $\mu \mathrm{M})$ or an anti-PAR1 antibody $(1: 100)$ and placed onto fibrinogen-coated Millicell glass slides for 1 hour at $37^{\circ} \mathrm{C}$. Adherent platelets were fixed with $4 \%$ paraformaldehyde, permeabilized with $0.1 \%$ Triton X-100, blocked with $5 \%$ bovine serum albumin and then stained with TRITC-labeled phalloidin at room temperature for 30 minutes. Fluorescence images were obtained on a confocal microscope (Zeiss Axio Observer LSM780, Oberkochen, Germany). The number of platelet adhesion events and the platelet spreading surface area were determined using NIH ImageJ software (NIH, Bethesda, MD, USA).

\section{Determination of Platelet Aggregation}

Agonist-induced platelet aggregation was measured using the PL-16 aggregometer (Sinnowa, Jiangsu, China) at $37^{\circ} \mathrm{C}$ and a constant stirring speed of $50 \mathrm{~g}$ to analyze platelet function. Briefly, platelets were incubated with thrombin $(0.5 \mathrm{U} / \mathrm{mL})$, Tp0136 $(10 \mu \mathrm{g} / \mathrm{mL})$, Tp17 $(10 \mu \mathrm{g} / \mathrm{mL})$ or PBS with or without different inhibitors or antagonists (RWJ56110, U73122 or PTX) for 5 minutes at $37^{\circ} \mathrm{C}$ and assessed on an aggregometer according to the manufacturer's protocol.

\section{Platelet-Mediated Clot Retraction Assay}

The platelet clot retraction experiment was performed as described by Ren et al. (17). After incubation with $10 \mu \mathrm{g} / \mathrm{mL}$ Tp0136 and Tp17 (PBS as a control), platelets were stimulated with $20 \mu \mathrm{g} / \mathrm{mL}$ fibrinogen and $0.5 \mathrm{U} / \mathrm{mL}$ thrombin and recorded at the indicated time point using a camera. The clot area was quantified based on the ratio of the clot area to the platelet suspension area at different time points using ImageJ software (National Institute of Mental Health, Bethesda, MD, USA).

\section{Platelet Activation Assays}

Measurement of the platelet surface molecules P-selectin $(18,19)$ and PF4 (20) as indices of platelet activation was performed by flow cytometry (BD FACSCanto II, NJ, USA) and the enzymelinked immunosorbent assay (ELISA). For the P-selectin measurement, platelets were diluted to $2.0 \times 10^{8} / \mathrm{mL}$ with modified Tyrode's buffer and incubated for 15 minutes with thrombin $(0.5 \mathrm{U} / \mathrm{mL}), \mathrm{Tp} 0136(10 \mu \mathrm{g} / \mathrm{mL})$ or PBS in the absence or presence of different antagonists or agonists. The aliquots (100 $\mu \mathrm{L})$ were then stained with an APC-labeled anti-human CD41 antibody (Biolegend, Shanghai, China) as a platelet identifier and with a FITC-labeled anti-human P-selectin antibody (Biolegend, Shanghai, China) before being analyzed by flow cytometry. Data were analyzed with FlowJo (TreeStar Software, Ashland, OR, USA). The levels of PF4 in PRP samples were assessed by ELISA (Human PF4 Simple Step ELISA ${ }^{\circledR}$ Kit, Abcam, MA, USA).

\section{cAMP Release Assays}

Cyclic adenosine monophosphate (cAMP) in PRP samples was assessed by a competition-based assay (cAMP ELISA Detection Kit, GenScript, NJ, USA). The PRP samples were preincubated with iloprost (final concentration, $100 \mathrm{ng} / \mathrm{mL}$ ) for 2 minutes, after which $10 \mu \mathrm{g} / \mathrm{mL}$ Tp0136 (PBS as a control) and RWJ56110 (1 $\mu \mathrm{M})$ were added alone or in combination. The samples were incubated for 15 minutes at $37^{\circ} \mathrm{C}$ and then analyzed for cAMP content.

\section{Determination of $\mathrm{Ca}^{2+}$ Fluxes}

The kinetics of intracellular $\mathrm{Ca}^{2+}$ mobilization were assessed as previously described (21). Platelets diluted in modified Tyrode's buffer $\left(2.0 \times 10^{8} / \mathrm{mL}\right)$ were incubated in Fluo-3-AM solution (Sigma, MO, USA) for 30 minutes at $37^{\circ} \mathrm{C}$. After determining the basal $\mathrm{Ca}^{2+}$ levels, Tp0136 $(10 \mu \mathrm{g} / \mathrm{mL})$ was added to the tube in the absence or presence of RWJ56110 $(1 \mu \mathrm{M})$, and the samples were assayed immediately. Flow cytometric analysis was then performed (BD FACSCanto II, NJ, USA).

\section{Cell Culture, Plasmid Cloning and Transfection}

Chinese hamster ovary (CHO) cells were cultured in DMEM supplemented with fetal bovine serum $(10 \% \mathrm{vol} / \mathrm{vol})$, penicillin $(100 \mathrm{U} / \mathrm{mL})$ and streptomycin $(100 \mu \mathrm{g} / \mathrm{mL})$. Full-length humanPAR1 cDNA was amplified and cloned into a pcDEF3-CMV-T7tagged vector (MiaoLingBio, Wuhan, China) to obtain a pcDEF3/PAR1 T7-tagged wild-type plasmid that was used to generate all mutants. pcDEF3 vectors encoding the T7-tagged PAR1 mutants L38S, D39S, P40N, R41A, S42D, and F43R were generated as described previously (22). CHO cells were transiently transfected with pcDEF3/PAR1 T7-tagged wild-type or PAR1 mutants using Lipofectamine 3000 Reagent (Invitrogen, Carlsbad, CA, USA) according to the manufacturer's recommendations and assessed by flow cytometry.

\section{Western Blotting Assays}

Platelets were stimulated with Tp0136 at different concentrations, and the PAR1 protein levels were measured by western blot as described previously (22). Platelets were treated with thrombin receptor activating peptide (TRAP)-6 $(10 \mu \mathrm{M})$ or Tp0136 $(10 \mu \mathrm{g} / \mathrm{mL})$ in the absence or presence of RWJ56110 (1 $\mu \mathrm{M})$ for 15 minutes at $37^{\circ} \mathrm{C}$. The cell lysates were collected, and the protein levels of phosphorylated and total PI3K, Akt, and p38 were detected by western blotting (23). Antibodies against PAR1 and PI3K/Akt/p38 signaling pathway components were purchased from Cell Signaling Technology (Danvers, MA, USA) or R\&D Systems (Minneapolis, MN, USA).

\section{Data Analysis and Statistics}

All results are expressed as the mean \pm standard error of the mean (SEM). Multiple groups were compared by one-way analysis of variance (ANOVA). Comparisons between two groups were made using paired Student's t-tests. Differences in calcium levels over time were assessed by repeated-measures ANOVA followed by Dunnett's post hoc test. All calculations were performed with the GraphPad Prism 6.0 program (version 5.0, GraphPad Software Inc, San Diego, CA, USA) and IBM SPSS statistics version 26 (SPSS, Inc., Chicago, IL, USA). A two-tailed $P$ value of $<0.05$ was considered statistically significant. 


\section{RESULTS}

\section{Tp0136 Promoted Human \\ Platelet Adhesion and Aggregation}

To elucidate the effect of Tp0136 on platelet adhesion and aggregation, platelets were treated with the Tp0136 protein, Tp17 protein, PBS (as a blank control) and thrombin (as a positive control). Platelet staining with TRITC-phalloidin revealed that the number of platelets adhered to the precoated fibrinogen in the Tp0136 group was higher than that in the PBS group $(P<0.001)$, Tp17 group was no change (Figure 1A). The platelet spreading surface area was assessed using NIH ImageJ software, and the average diffusion area of Tp0136-treated platelets immobilized on fibrinogen was markedly increased compared with that of PBS-treated platelets $(P<0.001)$, and Tp17-treated platelets was no statistical difference (Figure 1B). The effect of Tp0136 on platelet adhesion to fibrinogen was confirmed by the significantly higher adhesion of Tp0136stimulated platelets to the fibrinogen-coated surface as determined by ELISA $(P<0.001)$, compared with that of PBStreated platelets, and platelet adhesion was no change stimulated by Tp17 (Figure 1C). The platelet aggregation assay (Figure 1D) revealed an increased response to Tp0136-treated platelets $(P<0.001)$ and no increased in the Tp17-treated platelets. In addition, the result of the clot retraction assay showed that Tp0136-treated human platelets accelerated platelet aggregation

A
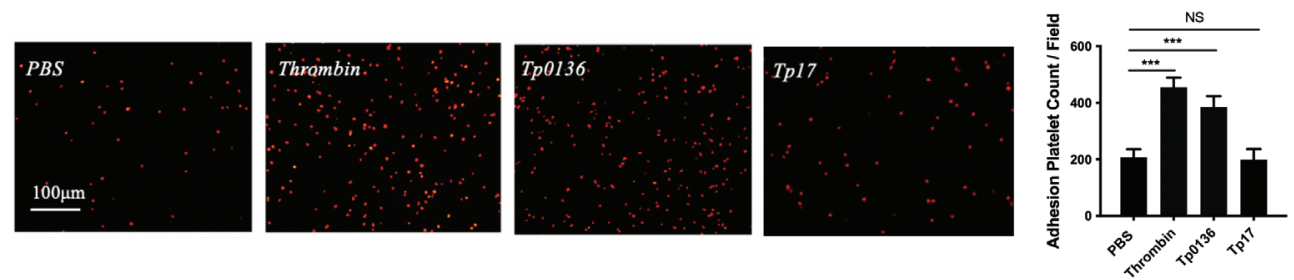

B
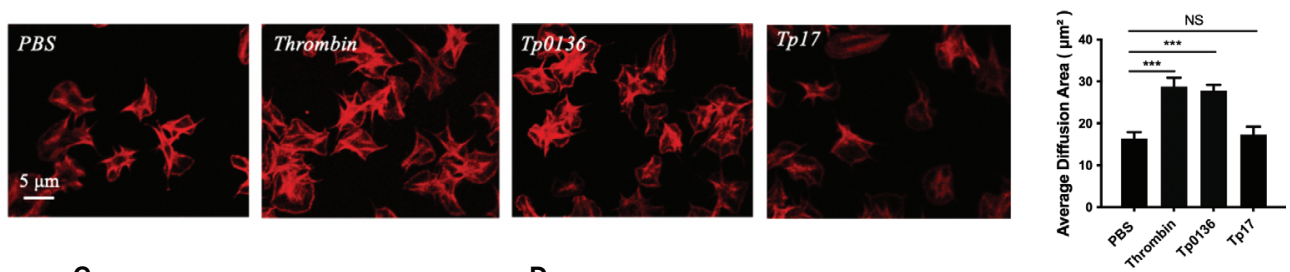

C

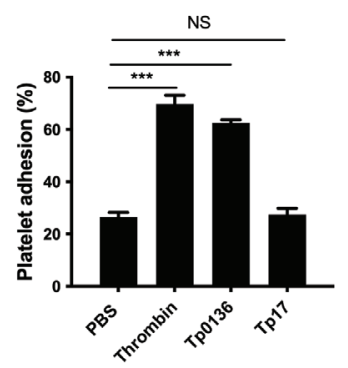

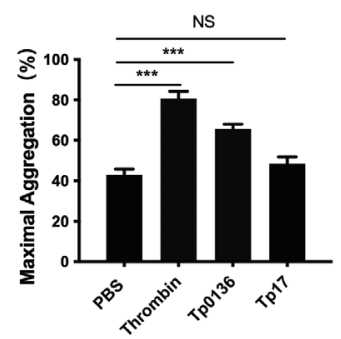

E
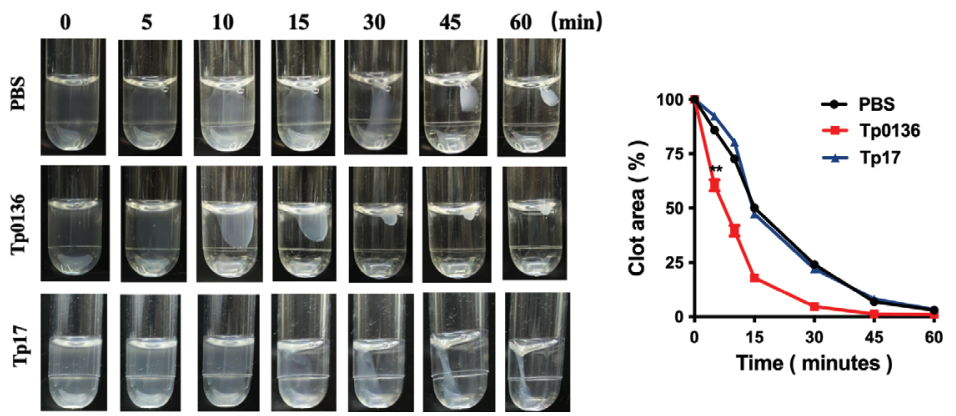

FIGURE 1 | Tp0136 promoted human platelet adhesion and aggregation. (A, B) Effect of Tp0136 on platelet adhesion as determined by staining with TRITC-labeled phalloidin. Statistical data were determined based on the number of platelet adhesions (A) and were calculated from the mean of the average surface area of individual platelets (B). (C) Platelet adhesion as assessed by ELISA. (D) The maximum aggregation rate of platelets was determined by an aggregometer. (E) Platelet clot retraction was quantified by the ratio of the clot area to the platelet suspension area at different time points. The values are presented as the mean \pm SEM of experimental triplicates and are representative of the results of three independent experiments. Values among multiple groups were compared by one-way ANOVA. Comparisons between two groups in the platelet clot retraction experiment were made using a paired t-test (NS, no significance, ${ }^{\star \star} P<0.01,{ }^{\star \star \star} P<0.001$ ). 
and that the aggregation area was significantly lower than that in the PBS group after 5 minutes $(P<0.01)$. The Tp0136-treated human platelets almost formed a very small clot after 30 minutes, while these was no difference between Tp17-treated group and PBS group, as demonstrated in Figure 1E. These data indicated that Tp0136 promoted human platelet adhesion and aggregation.

\section{Tp0136 Promoted Human Platelet Activation}

Given the promotional effect of Tp0136 on platelet adhesion and aggregation, we next investigated whether Tp0136 affects platelet activation. The flow cytometry results showed that the surface expression of P-selectin was increased in the Tp0136-treated platelet group by threefold compared with that in the PBS group $(P<0.001)$ (Figure 2A). In addition, Tp0136 significantly promoted the secretion of PF4 (vs. PBS-treated group, $P<0.01$ ) (Figure 2B). These data indicated that Tp0136 promoted platelet activation.

\section{Tp0136 Promoted Platelet Activation and Aggregation Through PAR1}

Human platelets express PAR1, and the activation of PAR1 is sufficient to trigger platelet secretion and aggregation (7). To confirm the regulation of platelet PAR1 receptors by Tp0136, platelets were treated with Tp0136 at different concentrations. The protein expression of PAR1 was decreased after treatment with Tp0136 at a concentration of $5 \mu \mathrm{g} / \mathrm{mL}(P<0.01)$, and the best response was achieved with $15 \mu \mathrm{g} / \mathrm{mL}$ Tp0136 $(P<0.01)$ (Figure 3A), indicating a concentration-dependent pattern. In addition, the pretreatment of platelets with RWJ56110 (a specific PAR1 antagonist) or an anti-human-PAR1 antibody ameliorated the activation and aggregation of platelets induced by Tp0136. RWJ56110 and the human-PAR1 antibody significantly reduced the expression of $\mathrm{P}$-selectin on platelets $(P<0.001)$ (Figure 3B), the secretion of PF4 $(P<0.05)$ (Figure 3C), platelet aggregation $(P<0.001)$ (Figure 3D), platelet adhesion $(P<0.001)$ (Figures 3E, F) and the average platelet diffusion area $(P<0.01)$ (Figure $3 \mathbf{G})$. Taken together, these results demonstrate that PAR1 is essential for the Tp0136-mediated promotion of platelet activation and aggregation.

Serine proteases, such as plasmin, thrombin, and activated protein $\mathrm{C}$, hydrolyze PAR1 to generate a tethered ligand that, in turn, activates PAR1 by interacting with the body of the receptor, thus triggering transmembrane signaling $(7,24,25)$. In our study, the cleavage of platelet PAR1 by Tp0136 was confirmed in $\mathrm{CHO}$ cells expressing the T7-tagged wild-type PAR1 plasmid (Figure 3H). To identify the specific cleavage site of Tp0136, we performed site-directed mutations of the key residues of the PAR1 N-terminus between amino acids 38 and 43, generating the T7-tagged PAR1 mutants L38S, D39S, P40N, R41A, S42D, and F43R (Figure 3I), and expressed them in CHO cells. The cleavage of PAR1 by Tp0136 was suppressed in T7-S42D PAR1transfected cells compared with T7-wild-type PAR1-transfected cells (Figure 3J). Thus, Tp0136 cleaves PAR1 at LDPR ${ }^{41} \downarrow S^{42} \mathrm{FL}$ to generate the $\mathrm{S}^{42}$ FLLRN-tethered ligand (TRAP-6), similar to that produced by thrombin (22).

\section{Tp0136 Enhanced $G_{q}$ Signaling Through PAR1 During Platelet Activation}

Activated PAR1 can couple with members of the $G_{q}$ and $G_{i}$ families and trigger numerous intracellular signaling pathways (8). To determine whether Tp0136 activated $\mathrm{G}_{\mathrm{q}}$-protein-coupled pathways through PAR1 to promote platelet activation and aggregation, the expression levels of Akt, PI3K and P38 were detected. As shown in Figure 4A, Tp0136 triggered the phosphorylation of Akt $(P<0.01)$, indicating $\mathrm{PI} 3 \mathrm{~K}$ activation $(P<0.01)$, and the phosphorylation of p38 $(P<0.01)$, confirming $\mathrm{G}_{\mathrm{q}}$-dependent signaling, similar to TRAP-6. To determine whether Tp0136 enhances $\mathrm{G}_{\mathrm{q}}$ signaling through PAR1, the PAR1 antagonist RWJ56110 was utilized. Interestingly, the RWJ56110 treatment of platelets significantly reduced the activation ability of Akt, PI3K and p38 compared with that of the conditioned media-treated controls, reversing the changes caused by Tp0136 (Figure 4A). In addition, Tp0136 significantly elicited a $\mathrm{G}_{\mathrm{q}}$-triggered increase in intraplatelet calcium levels $(P<0.05)$, as measured using the Fluo-3AM calcium indicator, and this effect was inhibited by the PAR1 antagonist
A

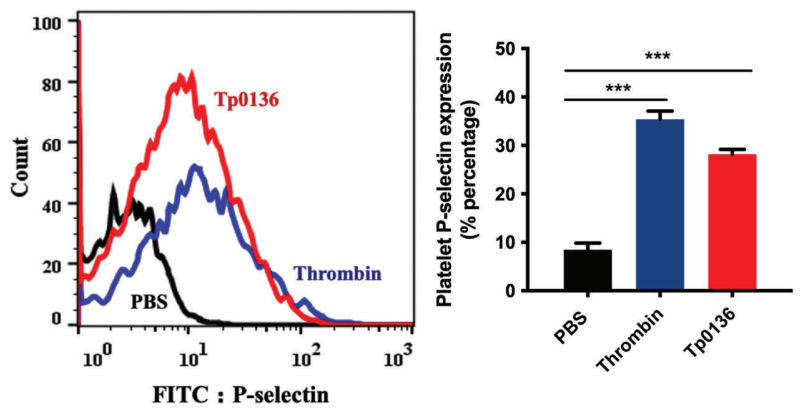

B

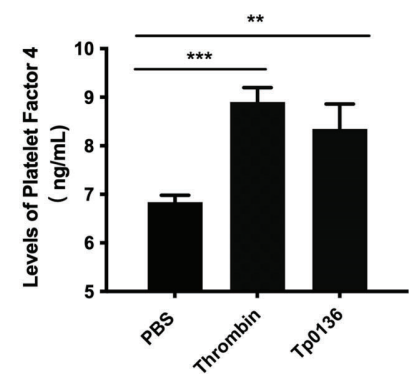

FIGURE 2 | Tp0136 promoted human platelet activation. (A) Expression of platelet P-selectin as measured by flow cytometry. A representative histogram is shown. Statistical data were analyzed using the X geometric mean fluorescence (left) and the percentage of gated cells (right). (B) PF4 expression as determined by ELISA. The values are presented as the mean \pm SEM of experimental triplicates and are representative of the results of three independent experiments. Values among multiple groups were compared by one-way ANOVA $\left({ }^{\star \star} P<0.01,{ }^{\star \star \star} P<0.001\right)$. 
A

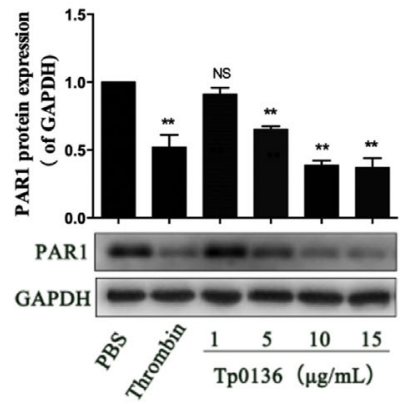

C

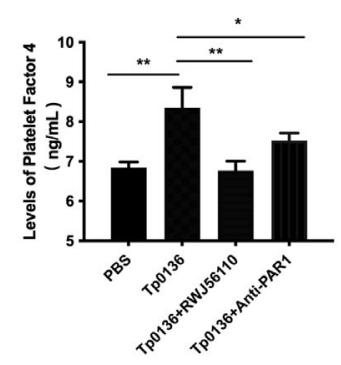

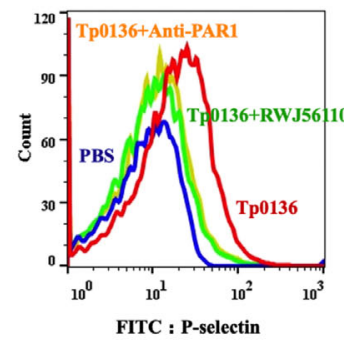

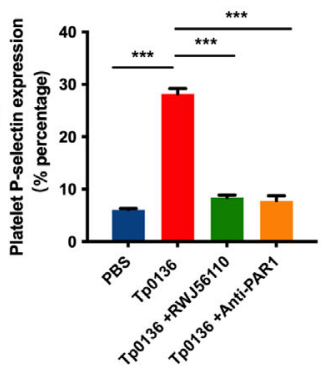

D

E

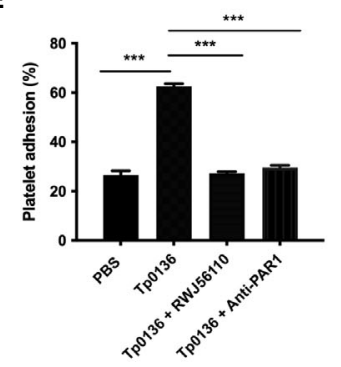

$\mathbf{F}$
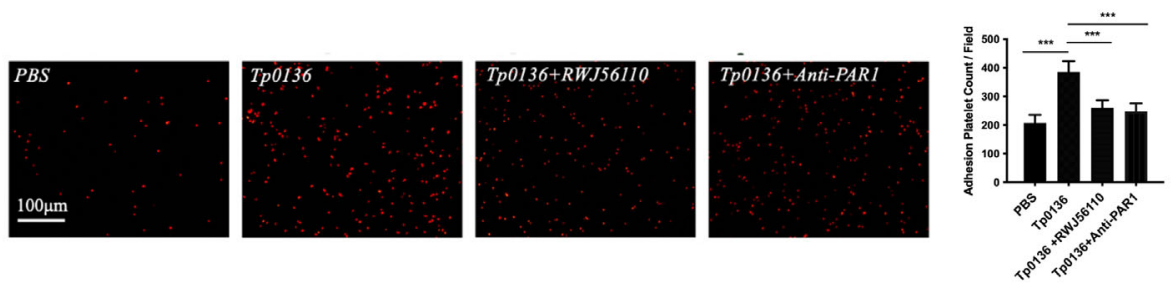

G
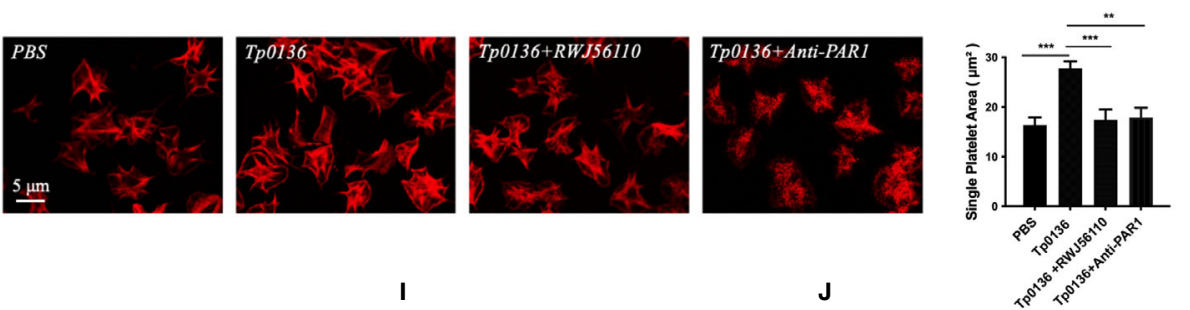

H

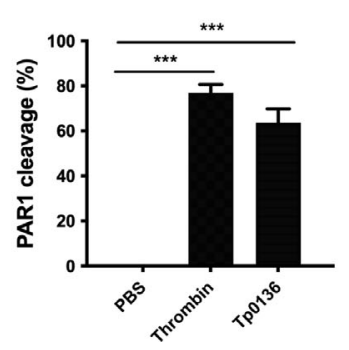

I

Thrombin

$\mathbf{J}$

humanPAR1 WT : -ESKATNATLDPRSFLLRNPND-L38S : -ESKATNATSDPRSFLLRNPND-D39S : -ESKATNATLSPRSFLLRNPND--

Mutants P40N : -ESKATNATLDNRSFLLRNPND--

R41A： -ESKATNATLDPASFLLRNPND--

S42D : -ESKATNATLDPRDFLLRNPND--

F43R : -ESKATNATLDPRSRLLRNPND--

TRAP-6 : SFLLRN

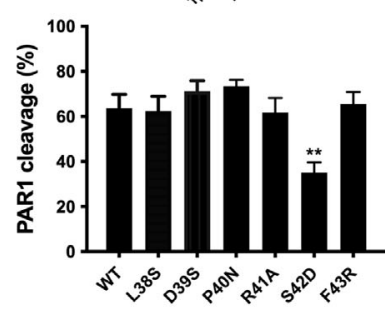

FIGURE 3 | Tp0136 promoted platelet activation and aggregation through PAR1. (A) PAR1 protein expression as determined by western blotting. (B-G) Effects of the PAR1 antagonist RWJ56110 and the anti-PAR1 antibody on platelet activation and aggregation induced by Tp0136. (B) P-selectin expression as measured by flow cytometry. (C) PF4 expression as measured by ELISA. (D) Maximal platelet aggregation as determined by an aggregometer. (E) Platelet adhesion as assessed by ELISA. (F,G) Platelet adhesion as assessed by staining with TRITC-labeled phalloidin. Statistical data are based on the number of platelet adhesions $(\mathbf{F})$ and were calculated from the mean of the average surface area of individual platelets (G). (H) Effect of Tp0136 on PAR1 as determined by flow cytometry. (I) Amino acid sequences of WT (wild-type) PAR1, PAR1 proteins with mutations in the extracellular domain and a PAR1 peptide agonist (TRAP-6). (J) Exploration of the Tp0136 protein sites that act on PAR1. The values are presented as the mean \pm SEM of experimental triplicates and are representative of the results of three independent experiments. Values among multiple groups were compared by one-way ANOVA. Comparisons between two groups were made using a paired t-test (NS, no significance, $\left.{ }^{\star} P<0.05,{ }^{\star \star} P<0.01,{ }^{\star \star \star} P<0.001\right)$. 
A
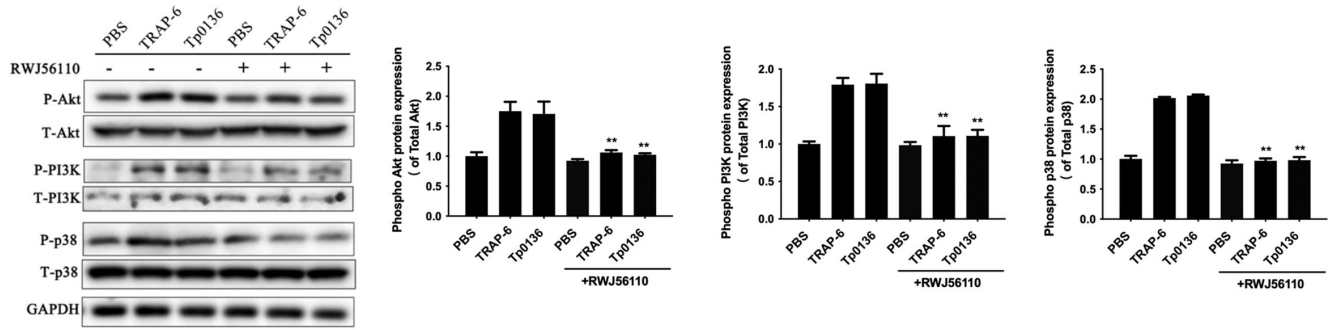

B
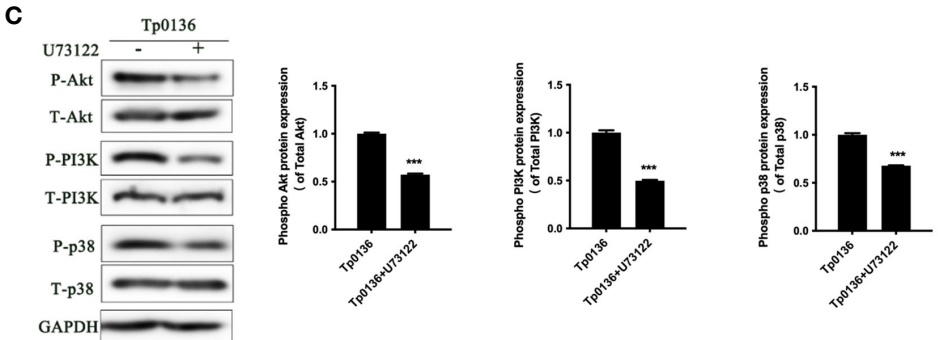

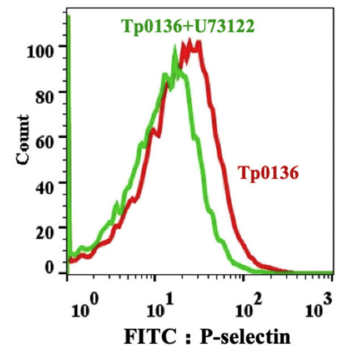

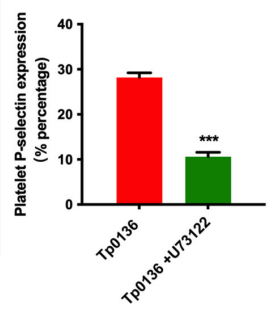

$\mathbf{E}$

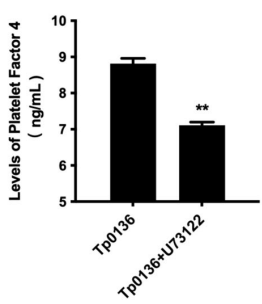

$\mathbf{F}$

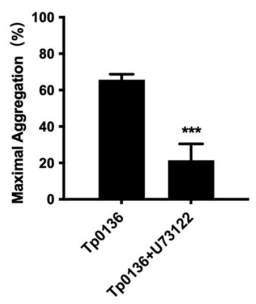

FIGURE 4 | Tp0136 enhanced $G_{q}$ signaling through PAR1 during platelet activation. (A) The protein expression of phosphorylated/total p38/Akt/PI3K and GAPDH was assessed by western blotting. (B) Cytosolic free $\mathrm{Ca}^{2+}$ was measured by flow cytometry. (C) The protein expression of phosphorylated/total p38/Akt/PI3K and GAPDH was assessed by western blotting. (D) Expression of platelet P-selectin as measured by flow cytometry. A representative histogram is shown. (E) PF4 as determined by ELISA. (F) Maximal platelet aggregation as determined by an aggregometer. The values are presented as the mean \pm SEM of experimental triplicates and are representative of the results of three independent experiments. Comparisons between the two groups were analyzed using a paired t-test. Differences in the calcium levels over time were determined by repeated-measures ANOVA followed by Dunnett's post hoc test $\left({ }^{*} P<0.05,{ }^{* \star} P<0.01,{ }^{* \star *} P<0.001\right)$.

RWJ56110 ( $P<0.05)$ (Figure 4B). The involvement of $\mathrm{G}^{\mathrm{q}}$-dependent signaling during Tp0136-induced platelet activation was significantly inhibited by U73122, a phospholipase C inhibitor $(P<0.001)$ (Figure 4C). The inhibitor U73122 significantly abolished the potentiating effect of Tp0136 on P-selectin expression $(P<0.001)$ (Figure 4D), the secretion of PF4 $(P<0.01)$ (Figure 4E) and platelet aggregation $(P<0.001)$ (Figure 4F).

\section{Tp0136 Enhanced $G_{i}$ Signaling Through PAR1 During Platelet Activation}

Furthermore, cAMP was measured to determine whether the effects of Tp0136 on platelets involve $\mathrm{G}_{\mathrm{i}}$-dependent signaling pathways. The iloprost-induced increase in the intraplatelet cAMP concentration was affected by Tp0136 $(P<0.05)$, and pretreatment with RWJ56110 increased the cAMP level compared to that in the group stimulated with only Tp0136 $(P<0.05)$ (Figure 5A). To assess the contribution of $G_{i}$ signaling to the activation of platelets by Tp0136, pertussis toxin (PTX), an inhibitor of the $G_{i}$ signaling pathway, was utilized to specifically inhibit $G_{i}$. PTX significantly reversed the enhancing effect of Tp0136 on platelet surface $\mathrm{P}$-selectin expression $(P<0.001)$ (Figure 5B) and PF4 granular secretion $(P<0.01)$ (Figure 5C) and weakened the enhancement of platelet aggregation induced by Tp0136 $(P<0.01)$ (Figure 5D), showing that $\mathrm{G}_{\mathrm{i}}$-dependent signaling was involved in the activating effect of Tp0136 on platelets.

\section{DISCUSSION}

The well-recognized capacity of T. pallidum, the etiological agent of venereal syphilis, for early dissemination and immune evasion has earned it the designation of 'the stealth pathogen' (26). Patients with primary syphilis present with typical chancres, painless ulcerations, that heal spontaneously over several weeks, which gives the illusion that the person has not been infected with syphilis and thus causes the best treatment period to be missed; thereafter, T. pallidum enters a latent state, inducing an 


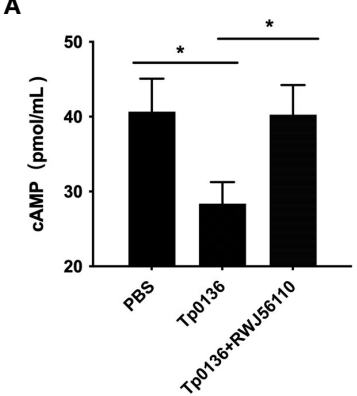

C

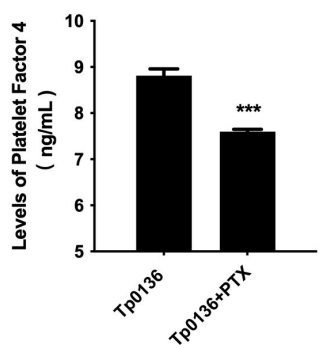

B
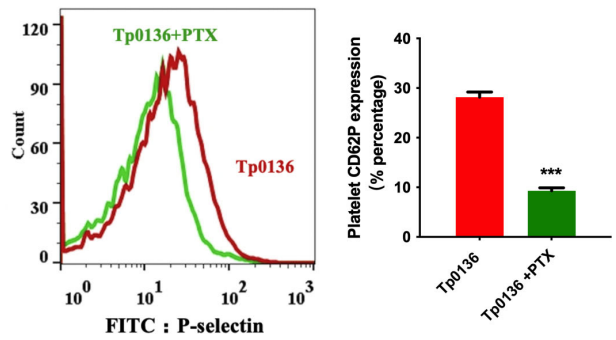

D

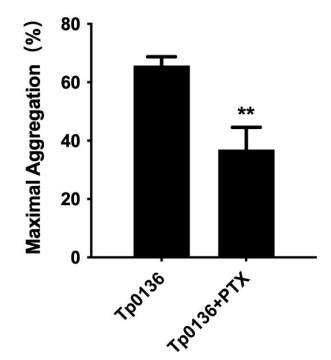

FIGURE 5 | Tp0136 enhanced $G_{i}$ signaling through PAR1 during platelet activation. (A) cAMP as analyzed by ELISA. (B) P-selectin as measured by flow cytometry. A representative histogram is shown. (C) PF4 as measured by ELISA. (D) Maximal platelet aggregation as determined by an aggregometer. The values are presented as the mean \pm SEM of experimental triplicates and are representative of the results of three independent experiments. Comparisons between the two groups were analyzed using a paired t-test $\left({ }^{\star} P<0.05,{ }^{\star \star} P<0.01,{ }^{\star \star \star} P<0.001\right)$.

insidious infection. Self-healing of chancre is essentially wound healing, which is an important multifaceted and complicated process in humans and animals that is governed by sequential but overlapping phases, including the hemostatic, inflammatory, proliferative, and remodeling phases (27). It is important to understand the first signals that activate the cellular response of injured tissue. After injury to the skin, the exposed subendothelial, collagen and tissue factors activate platelet aggregation, which results in degranulation and the release of chemotactic and growth factors to form the clot that initiates wound healing (28). In the present study, we found that Tp0136, a predicted $T$. pallidum adhesin that mediates its colonization during infection (9), enhanced platelet activation and aggregation through PAR1 and then initiated the receptor $G_{q}$ and $G_{i}$ signaling pathways. This process may represent the first step of platelet recruitment to syphilitic lesion sites and the initiation of wound healing. However, a recently research by Cameron group reported that T. pallidum directly, preferentially, and reversibly interacted with platelets, altered their movement and increased blood-brain barrier permeability, eventually facilitating their dissemination (14). Tp0136 as an important adhesion protein of $T$. pallidum, here we found that it could stimulate platelet activation and aggregation. Therefore, Tp0136 protein could play an important role in interaction with platelet mediating the dissemination of $T$. pallidum, which would need more research.

In our study, Tp0136 activated platelets and promoted platelet-fibrinogen adhesion and aggregation, resulting in the formation of platelet clots, which are naturally involved in wound healing (29). Upon activation, platelets secrete more than 300 active substances from their intracellular particles. Herein, Tp0136 activated platelets and promoted the platelet secretion of granules, such as PF4, P-selectin, and $\mathrm{Ca}^{2+}$, into the surrounding cellular milieu. These secreted platelet granule components contribute to blood coagulation (30). P-selectin is an inflammatory coagulation biomarker involved in clotting (31). Our results showed that Tp0136 promoted the expression of $\mathrm{P}$-selectin in platelets and promoted platelet activation and aggregation, which may have initiated the self-healing of chancres in the early stages of syphilis. Of course, this phenomenon needs to be further studied in animals (in vivo) infected with syphilis.

PARs are G-protein-coupled receptors that utilize a fascinating mechanism to convert an extracellular proteolytic cleavage event into a transmembrane signal; these receptors carry their own ligands, which remain hidden until unmasked by receptor cleavage (8). PAR1, the prototype of this family, is activated when thrombin cleaves its amino-terminal extracellular domain at a specific site. This cleavage reveals a new $\mathrm{N}$-terminus that acts as a tethered ligand for intramolecular binding to the body of the receptor and thus affects transmembrane signaling (32). We observed that Tp0136 enhanced platelet activation and aggregation through PAR1. We next carried out site-directed mutagenesis of PAR1 and found that Tp0136 acted on the Nterminal extracellular domain of PAR1 between residues 41 and 42 (LDPR ${ }^{41} \downarrow S^{42} \mathrm{FL}$ ), which was consistent with thrombin. However, whether the Tp0136 protein has the characteristics of an active enzyme still needs to be determined. 


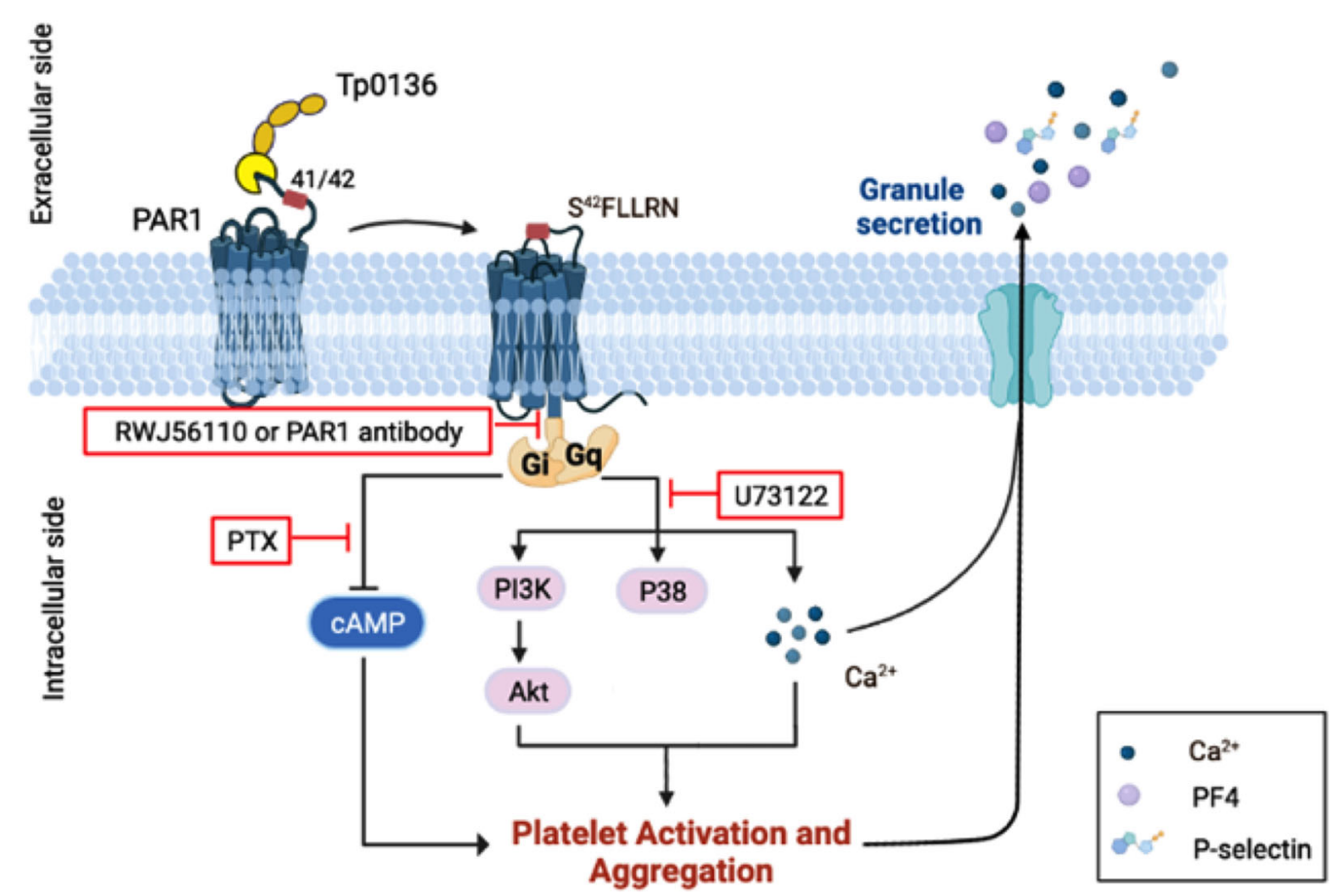

FIGURE 6 | Schematic model of the mechanism by which Tp0136 modulates platelet signaling. Tp0136 promotes human platelet activation and aggregation through PAR1 and generates an $S^{42} F L L R N$-tethered ligand that interacts with the receptor to induce PAR1 signaling. In particular, Tp0136 activates the G ${ }^{9}$ signaling pathway through PAR1, thereby inducing PAR1-dependent Akt-PI3K activation, p38 phosphorylation and $\mathrm{Ca}^{2+}$ flux, and the Gi pathway, thereby attenuating the release of cytosolic CAMP.

PAR1 can couple to members of the $\mathrm{G}_{\mathrm{q}}$ and $\mathrm{G}_{\mathrm{i}}$ families and thus to a host of intracellular effectors. Our results showed that Tp0136 enhanced platelet activation through PAR1, thereby initiating $\mathrm{G}_{\mathrm{q}^{-}}$and $\mathrm{G}_{\mathrm{i}^{-}}$activated intracellular pathways and thus predisposing platelets to be fully activated by a subsequent subthreshold stimulus. $G_{q}$ generates a pathway for calciumregulated kinases, mitogen-activated protein kinase cassettes, and other proteins that mediate cellular responses ranging from particle formation to integrin activation to platelet aggregation $(33,34)$. Furthermore, Akt is a serine/threoninespecific protein kinase that plays a key role in platelet aggregation, integrin signaling, particle secretion, and clot retraction (35). In our study, Tp0136 stimulated $\mathrm{G}_{\mathrm{q}}$ activation through PAR1 in human platelets, as shown by Akt-PI3K activation and p38 phosphorylation, and increased the release of intraplatelet $\mathrm{Ca}^{2+}$, a key second messenger, from intracellular stores $(36,37)$. As reported herein and in agreement with the findings of others (38), the treatment of platelets with U73122, a phospholipase $\mathrm{C}$ inhibitor, partially attenuated the upregulated expression of P-selectin and PF4 as well as the subsequent platelet adhesion and aggregation. These observations clearly underscore the critical regulatory effect of $\mathrm{Tp} 0136$ on the $\mathrm{G}_{\mathrm{q}}$ activated intracellular pathways downstream of PAR1. In addition, Tp0136 reduced the cAMP levels in iloprost-exposed platelets. Cytosolic cAMP is synthesized by adenylyl cyclase and is known as a powerful inhibitor of platelet aggregation (39).
Moreover, PTX, which inhibits $\mathrm{G}_{\mathrm{i}}$-receptor coupling pathways, affected the potentiating activity of Tp0136 on platelet aggregation. Based on the information discussed above, Tp0136 induces Akt-PI3K activation, p38 phosphorylation and $\mathrm{Ca}^{2+}$ movement in platelets; however, to achieve full platelet activation, Tp0136 must be induced by regulating the intracellular cAMP levels and thereby triggering the concomitant $G_{i}$ signaling pathway. A similar mechanism has been reported for other agents enhancing platelet activation, such as $\mathrm{PGE}_{2}$ (24) and MMP-2 (22), and explains the difference between platelet primers and full platelet agonists (40).

Several limitations should be noted. First, we showed that Tp0136 activated platelets to promote their aggregation in vitro, and further studies, such as in vivo experiments, is needed to confirm our in vitro findings. Second, we detected changes in the protein expression of only PAR1 and related downstream signaling molecules, and further study is needed to determine whether other platelet receptors are involved in this process. Third, the effect of Tp0136 promoting platelet activation and aggregation on the development of syphilis infection remains to be further studied.

In conclusion, were herein elucidated a mechanism of platelet activation and aggregation in which Tp0136 effects platelets through PAR1 and thereby triggers downstream $G_{i}$ and $G_{q}$ signaling (Figure 6). This study suggests that $\mathrm{Tp} 0136$ plays a role in platelet function, and the elucidation of relevant 
mechanisms represents another step toward understanding chancre self-healing in the early stages of syphilis.

\section{DATA AVAILABILITY STATEMENT}

The original contributions presented in the study are included in the article/supplementary material. Further inquiries can be directed to the corresponding author.

\section{ETHICS STATEMENT}

The studies involving human participants were reviewed and approved by the Ethics Committee of Zhongshan Hospital, Xiamen University. All volunteers provided written informed consent in accordance with the Declaration of Helsinki.

\section{AUTHOR CONTRIBUTIONS}

QY-X was first author. TC-Y was corresponding author. TC-Y and QY-X designed the study and drafted the manuscript, TC-Y and

\section{REFERENCES}

1. Tong ML, Lin LR, Liu LL, Zhang HL, Huang SJ, Chen YY, et al. Analysis of 3 Algorithms for Syphilis Serodiagnosis and Implications for Clinical Management. Clin Infect Dis (2014) 58(8):1116-24. doi: 10.1093/cid/ciu087

2. Peeling RW, Mabey D, Kamb ML, Chen XS, Radolf JD, Benzaken AS. Syphilis. Nat Rev Dis Primers (2017) 3:17073. doi: 10.1038/nrdp.2017.73

3. Rodrigues M, Kosaric N, Bonham CA, Gurtner GC. Wound Healing: A Cellular Perspective. Physiol Rev (2019) 99(1):665-706. doi: 10.1152/ physrev.00067.2017

4. Clark RA. Fibrin Is a Many Splendored Thing. J Invest Dermatol (2003) 121 (5):xxi-xxii. doi: 10.1046/j.1523-1747.2003.12575.x

5. Versteeg HH, Heemskerk JW, Levi M, Reitsma PH. New Fundamentals in Hemostasis. Physiol Rev (2013) 93(1):327-58. doi: 10.1152/physrev. 00016.2011

6. Sebert M, Sola-Tapias N, Mas E, Barreau F, Ferrand A. Protease-Activated Receptors in the Intestine: Focus on Inflammation and Cancer. Front Endocrinol (Lausanne) (2019) 10:717. doi: 10.3389/fendo.2019.00717

7. Kahn ML, Nakanishi-Matsui M, Shapiro MJ, Ishihara H, Coughlin SR. Protease-Activated Receptors 1 and 4 Mediate Activation of Human Platelets by Thrombin. J Clin Invest (1999) 103(6):879-87. doi: 10.1172/ JCI6042

8. Coughlin SR. Thrombin Signalling and Protease-Activated Receptors. Nature (2000) 407(6801):258-64. doi: 10.1038/35025229

9. Djokic V, Giacani L, Parveen N. Analysis of Host Cell Binding Specificity Mediated by the Tp0136 Adhesin of the Syphilis Agent Treponema Pallidum Subsp. Pallidum. PloS Negl Trop Dis (2019) 13(5):e0007401. doi: 10.1371/ journal.pntd.0007401

10. Ke W, Molini BJ, Lukehart SA, Giacani L. Treponema Pallidum Subsp. Pallidum TP0136 Protein Is Heterogeneous Among Isolates and Binds Cellular and Plasma Fibronectin via Its NH2-Terminal End. PloS Negl Trop Dis (2015) 9(3):e0003662. doi: 10.1371/journal.pntd.0003662

11. Luo X, Gao ZX, Lin SW, Tong ML, Liu LL, Lin LR, et al. Recombinant Treponema Pallidum Protein Tp0136 Promotes Fibroblast Migration by Modulating MCP-1/CCR2 Through TLR4. J Eur Acad Dermatol Venereol (2020) 34(4):862-72. doi: 10.1111/jdv.16162

12. Luo X, Lin SW, Xu QY, Ke WJ, Gao ZX, Tong ML, et al. Tp0136 Targets Fibronectin (RGD)/Integrin Betal Interactions Promoting Human
LR-L critical review and revision of the manuscript. QY-X and YJ-W performed experiments. LL-L was responsible for statistical analysis and validation. All authors agree to be accountable for the content of the work. All authors read and approved the final manuscript.

\section{FUNDING}

This work was supported by the National Natural Science Foundation of China (grant numbers 82172331, 81972028, 81973104, 81971147, 81772260, 81771312), the Key Projects for Province Science and Technology Program of Fujian Province, China (grant number 2020D017) and the Natural Science Foundation of Fujian Province, China (grant number 2021J02055). The funders played no role in the study design, data collection, or analyses, the decision to publish, or manuscript preparation.

\section{ACKNOWLEDGMENTS}

The authors thank all the contributors to this work.

Microvascular Endothelial Cell Migration. Exp Cell Res (2020) 396 (1):112289. doi: 10.1016/j.yexcr.2020.112289

13. Li QL, Tong ML, Liu LL, Lin LR, Lin Y, Yang TC. Effect of Anti-TP0136 Antibodies on the Progression of Lesions in an Infected Rabbit Model. Int Immunopharmacol (2020) 83:106428. doi: 10.1016/j.intimp.2020.106428

14. Church B, Wall E, Webb JR, Cameron CE. Interaction of Treponema Pallidum, the Syphilis Spirochete, With Human Platelets. PloS One (2019) 14(1):e0210902. doi: 10.1371/journal.pone.0210902

15. Boncler M, Kehrel B, Szewczyk R, Stec-Martyna E, Bednarek R, Brodde M, et al. Oxidation of C-Reactive Protein by Hypochlorous Acid Leads to the Formation of Potent Platelet Activator. Int J Biol Macromol (2018) 107(Pt B):2701-14. doi: 10.1016/j.ijbiomac.2017.10.159

16. Huang WC, Lin KC, Hsia CW, Hsia CH, Chen TY, Bhavan PS, et al. The Antithrombotic Agent Pterostilbene Interferes With Integrin Alphaiibbeta3Mediated Inside-Out and Outside-In Signals in Human Platelets. Int J Mol Sci (2021) 22(7):3643-58. doi: 10.3390/ijms22073643

17. Ren L, Li Q, You T, Zhao X, Xu X, Tang C, et al. Humanin Analogue, HNG, Inhibits Platelet Activation and Thrombus Formation by Stabilizing Platelet Microtubules. J Cell Mol Med (2020) 24(8):4773-83. doi: 10.1111/jcmm.15151

18. Berger GT, Hartwell DW. Wagner DD. P-Selectin and Platelet Clearance. Blood (1998) 92(11):4446-52. doi: 10.1182/blood.V92.11.4446

19. Théorêt JF, Yacoub D, Hachem A, Gillis MA. Merhi Y. P-Selectin Ligation Induces Platelet Activation and Enhances Microaggregate and Thrombus Formation. Thromb Res (2011) 128(3):243-50. doi: 10.1016/j.thromres. 2011.04 .018

20. Kaplan KL, Owen J. Plasma Levels of Beta-Thromboglobulin and Platelet Factor 4 as Indices of Platelet Activation. Vivo Blood (1981) 57(2):199-202. doi: 10.1182/blood.V57.2.199.199

21. Aliotta A, Bertaggia Calderara D, Alberio L. Flow Cytometric Monitoring of Dynamic Cytosolic Calcium, Sodium, and Potassium Fluxes Following Platelet Activation. Cytomet A (2020) 97(9):933-44. doi: 10.1002/cyto.a.24017

22. Sebastiano M, Momi S, Falcinelli E, Bury L, Hoylaerts MF, Gresele P. A Novel Mechanism Regulating Human Platelet Activation by MMP-2-Mediated PAR1 Biased Signaling. Blood (2017) 129(7):883-95. doi: 10.1182/blood2016-06-724245

23. Kim S, Jin J, Kunapuli SP. Relative Contribution of G-Protein-Coupled Pathways to Protease-Activated Receptor-Mediated Akt Phosphorylation in Platelets. Blood (2006) 107(3):947-54. doi: 10.1182/blood-2005-07-3040 
24. Fabre JE, Nguyen M, Athirakul K, Coggins K, McNeish JD, Austin S, et al. Activation of the Murine EP3 Receptor for PGE2 Inhibits cAMP Production and Promotes Platelet Aggregation. J Clin Invest (2001) 107(5):603-10. doi: 10.1172/JCI10881

25. Kuliopulos A, Covic L, Seeley SK, Sheridan PJ, Helin J, Costello CE. Plasmin Desensitization of the PAR1 Thrombin Receptor: Kinetics, Sites of Truncation, and Implications for Thrombolytic Therapy. Biochemistry (1999) 38(14):4572-85. doi: 10.1021/bi9824792

26. Radolf JD, Deka RK, Anand A, Šmajs D, Norgard MV, Yang XF. Treponema Pallidum, the Syphilis Spirochete: Making a Living as a Stealth Pathogen. Nat Rev Microbiol (2016) 14(12):744-59. doi: 10.1038/ nrmicro.2016.141

27. Wang PH, Huang BS, Horng HC, Yeh CC, Chen YJ. Wound Healing. J Chin Med Assoc (2018) 81(2):94-101. doi: 10.1016/j.jcma.2017.11.002

28. Gauglitz GG, Korting HC, Pavicic T, Ruzicka T, Jeschke MG. Hypertrophic Scarring and Keloids: Pathomechanisms and Current and Emerging Treatment Strategies. Mol Med (2011) 17(1-2):113-25. doi: 10.2119/ molmed.2009.00153

29. Heher P, Muhleder S, Mittermayr R, Redl H, Slezak P. Fibrin-Based Delivery Strategies for Acute and Chronic Wound Healing. Adv Drug Deliv Rev (2018) 129:134-47. doi: 10.1016/j.addr.2017.12.007

30. Golebiewska EM, Poole AW. Platelet Secretion: From Haemostasis to Wound Healing and Beyond. Blood Rev (2015) 29(3):153-62. doi: 10.1016/ j.blre.2014.10.003

31. Blann AD, Nadar SK, Lip GY. The Adhesion Molecule P-Selectin and Cardiovascular Disease. Eur Heart J (2003) 24(24):2166-79. doi: 10.1016/ j.ehj.2003.08.021

32. Vu TK, Hung DT, Wheaton VI, Coughlin SR. Molecular Cloning of a Functional Thrombin Receptor Reveals a Novel Proteolytic Mechanism of Receptor Activation. Cell (1991) 64(6):1057-68. doi: 10.1016/0092-8674(91) 90261-v

33. Adam F, Kauskot A, Nurden P, Sulpice E, Hoylaerts MF, Davis RJ, et al. Platelet JNK1 Is Involved in Secretion and Thrombus Formation. Blood (2010) 115(20):4083-92. doi: 10.1182/blood-2009-07-233932

34. Flevaris P, Li Z, Zhang G, Zheng Y, Liu J, Du X. Two Distinct Roles of Mitogen-Activated Protein Kinases in Platelets and a Novel Rac1-MAPKDependent Integrin Outside-in Retractile Signaling Pathway. Blood (2009) 113(4):893-901. doi: 10.1182/blood-2008-05-155978
35. O’Brien KA, Stojanovic-Terpo A, Hay N, Du X. An Important Role for Akt3 in Platelet Activation and Thrombosis. Blood (2011) 118(15):4215-23. doi: 10.1182/blood-2010-12-323204

36. Anderson R, Theron AJ, Steel HC, Nel JG, Tintinger GR. ADP-Mediated Upregulation of Expression of CD62P on Human Platelets Is Critically Dependent on Co-Activation of P2Y1 and P2Y12 Receptors. Pharmaceut (Basel) (2020) 13(12):420-35. doi: 10.3390/ph13120420

37. Koupenova M, Ravid K. Biology of Platelet Purinergic Receptors and Implications for Platelet Heterogeneity. Front Pharmacol (2018) 9:37:37. doi: 10.3389/fphar.2018.00037

38. Lopez JJ, Redondo PC, Salido GM, Pariente JA, Rosado JA. Two Distinct Ca2+ Compartments Show Differential Sensitivity to Thrombin, ADP and Vasopressin in Human Platelets. Cell Signal (2006) 18(3):373-81. doi: 10.1016/j.cellsig.2005.05.006

39. Nam GS, Nam KS. Arctigenin Attenuates Platelet Activation and Clot Retraction by Regulation of Thromboxane A2 Synthesis and cAMP Pathway. BioMed Pharmacother (2020) 130:110535. doi: 10.1016/j. biopha.2020.110535

40. Gresele P, Falcinelli E, Momi S. Potentiation and Priming of Platelet Activation: A Potential Target for Antiplatelet Therapy. Trends Pharmacol Sci (2008) 29(7):352-60. doi: 10.1016/j.tips.2008.05.002

Conflict of Interest: The authors declare that the research was conducted in the absence of any commercial or financial relationships that could be construed as a potential conflict of interest.

Publisher's Note: All claims expressed in this article are solely those of the authors and do not necessarily represent those of their affiliated organizations, or those of the publisher, the editors and the reviewers. Any product that may be evaluated in this article, or claim that may be made by its manufacturer, is not guaranteed or endorsed by the publisher.

Copyright (c) $2022 \mathrm{Xu}$, Wang, Lin, Liu and Yang. This is an open-access article distributed under the terms of the Creative Commons Attribution License (CC BY). The use, distribution or reproduction in other forums is permitted, provided the original author(s) and the copyright owner(s) are credited and that the original publication in this journal is cited, in accordance with accepted academic practice. No use, distribution or reproduction is permitted which does not comply with these terms. 